\title{
Taking the pulse of pulse oximetry in Africa
}

$\mathrm{T}$

he use of pulse oximetry for anesthesia monitoring during surgery has become the standard of care in the developed world.

Although a causal link between pulse oximetry and anesthesia death rates has not been firmly established, there is a consensus belief that ability to monitor the oxygenation of a sedated patient's hemoglobin improves the safety of anesthesia. Many believe it is a contributor to the dramatic reduction in anesthesia death rates over the course of recent decades, and argue that the proposition is supported by the fact that the reduction in anesthesia death rates hasn't been as rapid in the developing world, where the World Health Organization (WHO) has estimated that as many as seven million people will be disabled, and one million will die, annually as a result of complications from surgery.

In hopes of reducing that toll, WHO launched a Safe Surgery Saves Lives program in 2007 - complete with a minimum set and checklist of surgical safety standards - as well as a Global Pulse Oximetry Project in 2008, whose aims include the development of an inexpensive pulse oximeter for use in the developing world, where death rates in some countries are "100 to 1000 times higher" than in the developed world (www.who.int/patientsafety /events/08/1st_pulse_oximetry_meeting _background_doc.pdf).

There's no doubt that the need for pulse oximetry is particularly acute in sub-Saharan Africa, says Dr. Arthur Kwizera, an anesthesiologist at the Mulago Hospital in Kampala, Uganda. Pulse oximetry is expensive, he says. "A pulse oximeter monitor goes for up to $\$ 300 . "$

But Kwizera and a research team from the University of British Columbia believe they may be onto something that could solve the problem.

They're jointly involved in a pilot project in which a UBC-developed mobile phone-based pulse oximeter is

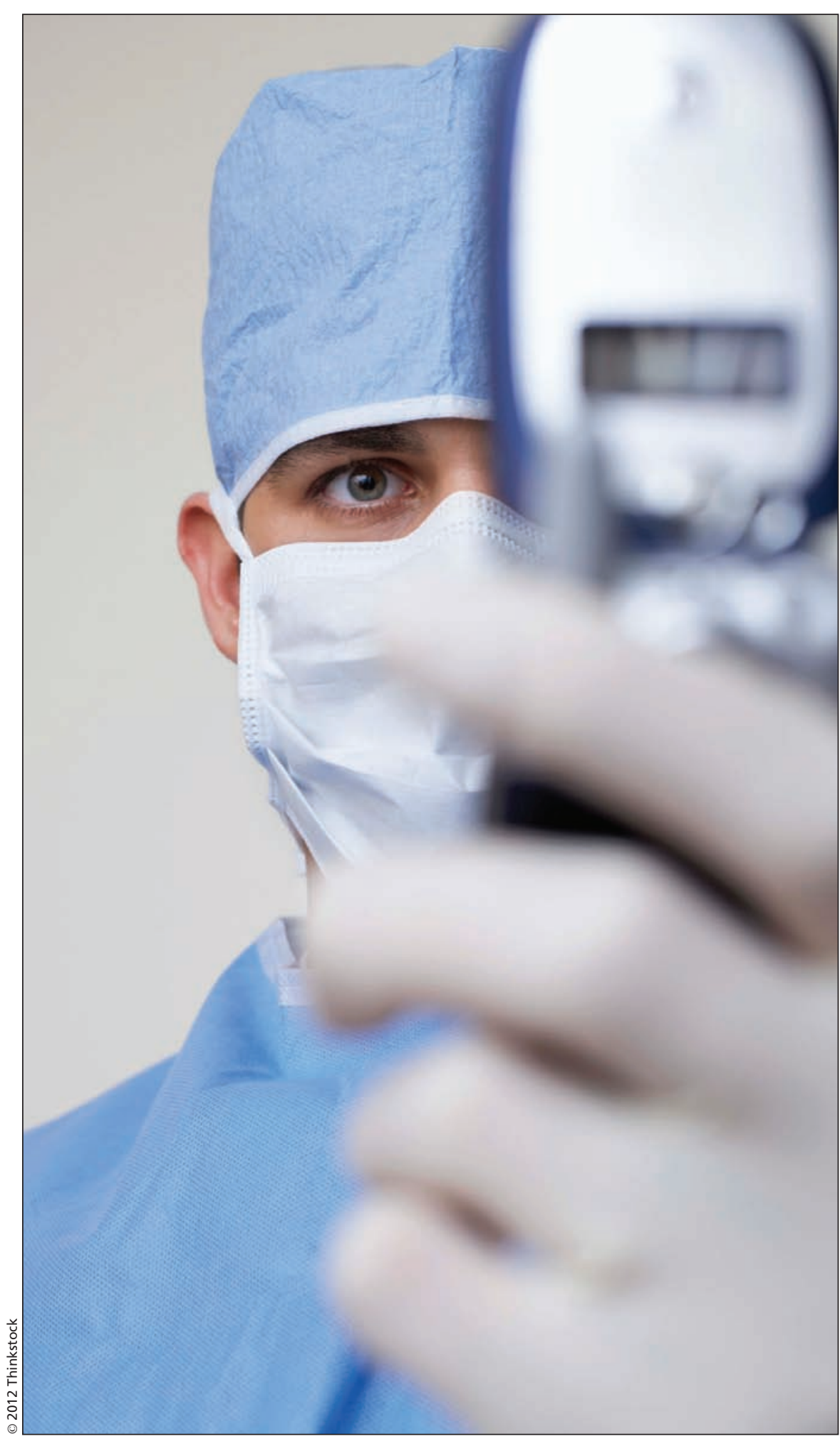

It is hoped a mobile phone-based pulse oximeter will be substantially more affordable in an African context. 
being tested at the Mulago Hospital as a potentially cheap means of providing pulse oximetry.

Adapting mobile phones into pulse oximeters makes them significantly more affordable in an African context, says Kwizera, who estimates that only $20 \%-40 \%$ of hospitals in Uganda have pulse oximeters.

If successful, the pilot has enormous ramifications in Africa, Kwizera adds. "What is the cheapest (electronic) thing in Africa? It's a mobile phone. Right now phones are being sold for $\$ 15$ and if you buy a $\$ 3$ patient probe. ... You can actually have a pulse oximeter on your phone for $\$ 20$, a tenth of what a $\$ 250$ pulse oximeter costs."

"This initiative, using a cell phone (as a pulse oximeter) leverages technology that has already made unbelievable strides in the developing world ... to move pulse oximetry forward," adds Dr.
Mark Ansermino, a lead researcher on the team that developed the device and an associate professor of anesthesiology at the University of British Columbia in Vancouver.

The "phone oximeter" uses smart phones but the pilot project aims to make it operational using lower-cost cell phones.

Mobile phones are ideal in the developing world because of their widespread availability and accessibility, says Ansermino. "There is really no other technology that has become so ubiquitous." They're easily adaptable, he adds. "Cell phones are amazingly powerful computing devices so we just had to find a way to get the software (for pulse oximetry) on to them."

The UBC team also hopes the oximeter might be ultimately modified for use in identifying women with a high risk of developing severe outcomes from preeclampsia. As envisioned within that context, it would use oxygen saturation and other factors such as maternal and gestational age and blood pressure to create a "score" that would predict which women are at higher risk, explains Dr. Peter von Dadelszen, an associate professor of obstetrics and gynecology at UBC. Pulse oximetry "is a reasonable predictor even by itself, but adding it in with other factors we have very good predictor."

Complications from preeclampsia are the second highest cause of maternal mortality worldwide, killing about 76000 women annually.

The team also hopes to soon launch a 1000-patient study to examine the device's utility in diagnosing childhood pneumonia. - Jocelyn Edwards, Kampala, Uganda

CMAJ 2012. DOI:10.1503/cmaj.109-4121 